\title{
Symbiont dynamics of the Tibetan tick Haemaphysalis tibetensis (Acari: Ixodidae)
}

\author{
Rongrong Wang ${ }^{1 \dagger}$, Ningxin $\mathrm{Li}^{1 \dagger}$, Jiannan $\mathrm{Liu}^{1}$, Tuo Li $i^{1}$, Ming Liu', Zhijun Yu${ }^{1,2^{*}}$ and Jingze $\mathrm{Liu}^{1 *}$
}

\begin{abstract}
Background: Characterization of the microbial diversity and symbiont dynamics of ticks may help to understand the development of ticks and reveal new strategies to control tick-transmitted pathogens, which has not yet been explored in the Tibetan tick Haemaphysalis tibetensis. This tick species is widely distributed in the Tibetan Plateau, and is recognized as one of the primary parasites affecting domestic and wild animals.

Methods: In the present study, the endosymbionts of $H$. tibetensis were characterized using diagnostic polymerase chain reaction (diagnostic PCR), and further evaluated for tissue distribution and population dynamics at each developmental stage of ticks and in tissues at different reproductive statuses by real-time quantitative polymerase chain reaction (RT-qPCR).

Results: Two symbionts were found in H. tibetensis, and named as CLS-Ht (Coxiella-like symbiont in H. tibetensis) and RLS-Ht (Rickettsia-like symbiont in H. tibetensis). They showed 100\% infection rate in both females and males of $\mathrm{H}$. tibetensis. CLS-Ht and RLS-Ht can be observed within eggs, larvae, nymphs and adults, which indicates vertical transmission in H. tibetensis. CLS-Ht was specifically distributed in the female ovaries and Malpighian tubules, whereas RLS-Ht was detected within ovaries, Malpighian tubules, salivary glands and midguts of the ticks. Real-time gPCR suggested that adult ticks carried the largest amount of CLS-Ht and RLS-Ht with CLS-Ht having a significantly higher presence in females than in males $(P<0.05)$, whereas the presence of RLS-Ht showed no significant differences between sexes. In the ovaries, CLS-Ht distribution reached a peak at one day post-engorgement, and then gradually declined to a lower level, whereas no change was observed in RLS-Ht. In Malpighian tubules, the amount of both symbionts displayed an increasing trend with time post-engorgement. In midguts and salivary glands, the amount of RLS-Ht showed no significant differences.

Conclusion: Two novel endosymbionts (CLS-Ht and RLS-Ht) were characterized in $\mathrm{H}$. tibetensis both showing a high prevalence and stable vertical transmission. The described tissue distribution and population dynamics might imply the important functions of these symbionts during the development and reproduction of ticks.
\end{abstract}

Keywords: Haemaphysalis tibetensis, Endosymbionts, Coxiella, Rickettsia, Tissue distribution, Population dynamics

\section{Background}

Ticks are obligate blood-sucking ectoparasites of many vertebrate animals and can transmit a diversity of pathogens including bacteria (rickettsiae and spirochetes), viruses and protozoans [1]. As the worldwide distribution and dynamic frequency from 'on-host' to 'off-host' changes, the involvement of ticks in commensal, mutualistic or parasitic

\footnotetext{
*Correspondence: zhijunyu@cunet.carleton.ca; liujingze@hebtu.edu.cn ${ }^{\dagger}$ Equal contributors

${ }^{1}$ Key Laboratory of Animal Physiology, Biochemistry and Molecular Biology of Hebei Province, College of Life Sciences, Hebei Normal University,

Shijiazhuang 050024, China

Full list of author information is available at the end of the article
}

interactions with different kinds of microorganisms becomes unavoidable $[2,3]$.

Many symbionts and complex bacterial communities have been explored from different tick species $[4,5]$, different tissues and organs [6,7], different life stages [8] and different feeding statuses [9]. These symbionts include Coxiella-like symbionts [10], Rickettsia-like symbionts, Arsenophonus-like symbionts [11], Francisella-like symbionts [12, 13], "Candidatus Midichloria mitochondrii" and Wolbachia-like symbionts [14]. Some microbes have been shown to provide the necessary nutrition needed for tick development whereas others have been shown to interfere 
with survival and transmission of tick-borne pathogens [15]. Among the tick-associated symbionts, Coxiella-like symbionts have been detected in several genera of ticks $[16,17]$, and were found mainly infecting ovaries and vertically transmitted by transovarial transmission $[18,19]$. Eliminating of Coxiella-like symbionts with antibiotics could cause severe reduction in fecundity and fitness of Amblyomma americanum [20], and recent genome studies on Coxiella-like symbionts in A. americanum and Rhipicephalus turanicus suggested their specific functions in providing required nutrients lacking in a blood meal $[21,22]$. Furthermore, Coxiella-like symbionts could impact the colonisation and transmission of other pathogens [16]. Similarly, Rickettsia-like symbionts characterized in Dermacentor variabilis and Dermacentor andersoni show little or no pathogenicity in laboratory animials, but can influence the physiology of host ticks and affect the transmission of the coinfected pathogenic rickettsiae [16]. A metabolic reconstruction on the genome of Rickettsia endosymbionts in both Ixodes scapularis and Ixodes pacificus has revealed the present of folate (B9 vitamin) biosynthesis genes [23]. Hence, characterizing the symbiont dynamics in ticks may help understand the development of ticks and reveal new strategies to control tick-transmitted pathogens.

The tick Haemaphysalis tibetensis is an important endemic-pathogen vector in the Qinghai-Tibet Plateau, from which new strains of spirochete and orbivirus were isolated $[24,25]$. This species of tick can occur above an altitude of 4,000 $\mathrm{m}$ where the environment is cold and dry [26]. The microbial diversity and symbiontic dynamics of $H$. tibetensis have not yet been explored, therefore, the current study investigated the endosymbionts in $H$. tibetensis, and the tissue tropism, population dynamics and vertical transmission of these endosymbionts were further evaluated in the hope of a better understanding the relationship between this species of tick and its different microorganisms.

\section{Methods}

\section{Collection and rearing of ticks}

Free-living $H$. tibetensis ticks were collected by flag dragging from vegetations in the Damxung County $\left(90^{\circ} 45^{\prime}-91^{\circ} 31^{\prime} \mathrm{E}, 29^{\circ} 31^{\prime}-31^{\circ} 04^{\prime} \mathrm{N}\right.$; altitude $\left.4,353 \mathrm{~m}\right)$, north Lhasa City, Tibet Autonomous Region, China, and identified according to available characteristics [27-29]. Part of the collected ticks (defined as field colony) were frozen in liquid nitrogen and then preserved at $-80{ }^{\circ} \mathrm{C}$ until use; others were reared on domestic rabbits Oryctolagus cuniculus as described previously [30]. Offspring of $H$. tibetensis (defined as laboratory colony) were maintained at $26{ }^{\circ} \mathrm{C}$, humidity $80 \%$ with a light: dark regime of $16: 8 \mathrm{~h}$.

\section{Dissection of ticks}

Ticks were first surface sterilized with $70 \%$ ethanol (3 washes) and then dissected sterilely under a stereomicroscope at $10 \times 23$ magnification using a micro-clipper in sterile phosphate-buffered saline (PBS) $\quad\left(\begin{array}{llllllll}137 & \mathrm{mM} & \mathrm{NaCl} & 2.7 & \mathrm{mM} & \mathrm{KCl} & 4.3 \mathrm{mM}\end{array}\right.$ $\mathrm{Na}_{2} \mathrm{HPO}_{4} \cdot 7 \mathrm{H}_{2} \mathrm{O}, 1.4 \mathrm{mM} \mathrm{KH_{2 }} \mathrm{PO}_{4}, \mathrm{pH}$ 7.4) as described previously [31]. Specific organs including ovaries, Malpighian tubules, salivary glands and midguts were separately collected in $1.5 \mathrm{ml}$ sterile vials (Axygen, Union City, USA) and frozen in $-80{ }^{\circ} \mathrm{C}$ for subsequent use.

\section{Genomic DNA extraction}

Total genomic DNA was extracted from each group of adults (10 females and 10 males, respectively) from field or laboratory colonies, and from dissected tissues and organs using a Genomic DNA isolation kit (Qiagen, Hilden, Germany). The concentration of the extracted genomic DNA was measured using a Nanodrop (Thermo Fisher Scientific, Waltham, USA) and the purity was evaluated by electrophoresis of the extracts in $1 \%(\mathrm{w} / \mathrm{v})$ agarose gels.

\section{Bacterial 16S rRNA gene library and restriction fragment length polymorphism (RFLP) analysis}

The bacterial 16S rRNA gene library was constructed using the genomic DNA of pooled ticks from field and laboratory colony. A $\sim 1,500$ bp fragment of 16S rRNA gene was amplified using bacterial universal primers Eub27F/Eub1492R [32] (Table 1). Resultant PCR products were purified with a PCR Purification Kit (Bioteke, Beijing, China) and ligated into the pEASY-T1 cloning vector using the pEASY-T1 simple cloning kit (TransGen, Beijing, China). Recombinant DNA was transformed into Escherichia coli TOP10 competent cells (TransGen, China). Thereafter, both Hae III and RsaI restriction endonucleases were applied to digest the gene library for subsequent RFLP analysis. Positive clones with different restriction fragment patterns were sequenced (Sangon Biotech, Shanghai, China) and blasted in the NCBI database (http://www.ncbi.nlm.nih.gov/BLAST/).

\section{Phylogenetic analysis}

The 1,500 bp 16S rRNA gene sequences obtained were compared with known sequences listed in the GenBank nucleotide sequence databases, aligned using the CLUSTAL W program, and manually checked with excluded DNA gaps. The phylogenetic trees were produced according to the neighbor-joining method after Kimura 2-parameter correction in the MEGA version 6 using bootstrap analyses with 1,000 replicates; the grampositive bacterium Bacillus subtilis (X60646) was used as the outgroup species. 
Table 1 Oligonucleotide primers used for PCR amplification and sequencing

\begin{tabular}{|c|c|c|c|c|c|c|}
\hline Primer & Species & Target gene & $\begin{array}{l}\text { Nucleotide sequence } \\
\left(5^{\prime}-3^{\prime}\right)\end{array}$ & $\begin{array}{l}\text { Annealing temperature } \\
\left({ }^{\circ} \mathrm{C}\right)\end{array}$ & $\begin{array}{l}\text { Approx. product size } \\
\text { (bp) }\end{array}$ & Reference \\
\hline CLS-F & Coxiella & $16 \mathrm{~S}$ rRNA & CACGTAGGAATCTACCTTGTAG & 55 & 90 & [4] \\
\hline CLS-R & & & CGTITGTTCCGAAGAAATTAT & & & \\
\hline Eub27F & Eubacteria & $16 \mathrm{~S}$ rRNA & AGAGTTTGATCCTGGCTCAG & 55 & 1,500 & {$[32]$} \\
\hline Eub1492R & & & TACCTTGTTACGACTT & & & \\
\hline Rickettsia354F & Rickettsia & $16 \mathrm{~S}$ rRNA & CAGCAATACCGAGTGAGTGATGAAG & 56 & 350 & [33] \\
\hline Rickettsia647R & & & AGCGTCAGTTGTAGCCCAGATG & & & \\
\hline Actin-F & Haemaphysalis & actin & CGTTCCTGGGTATGGAATCG & 55 & 100 & [4] \\
\hline Actin-R & Haemaphysalis & actin & TCCACGTCGCACTTCATGAT & & & \\
\hline
\end{tabular}

\section{Prevalence, tissue distribution and dynamic of the symbionts}

The prevalence, vertical transmission and dynamics of the symbionts were evaluated and monitored using SYBR green-based real-time qPCR. Briefly, genomic DNA was isolated from several groups including individual adults (50 females and 50 males) from the laboratory colony, and pooled samples of ticks in various developmental stages (500 eggs, 200 larvae and 50 nymphs, respectively), or from different organs. Standard curves were established by serial dilutions of plasmids containing inserts of the amplified 16S rRNA gene sequences from symbionts and host (tick) actins (Table 1). The $25 \mu \mathrm{l}$ master mix was composed of $12.5 \mu \mathrm{l}$ of $2 \times$ TransStart $^{\text {TM }}$ Top Green qPCR SuperMix (TransGen, China), $0.5 \mu \mathrm{l}$ of each $10 \mu \mathrm{M}$ primer [33] (Table 1), $10.5 \mu \mathrm{l} \mathrm{H}_{2} \mathrm{O}$ and $1 \mu \mathrm{l}$ template DNA. The qPCR assays were conducted in 96-well polypropylene plates in a Mx3005P qPCR system (Agilent Technologies, Santa Clara, USA) and conditions were set as follows: $94{ }^{\circ} \mathrm{C}$ for $30 \mathrm{~s} ; 40$ cycles of $94{ }^{\circ} \mathrm{C}$ for $5 \mathrm{~s}$ and $60{ }^{\circ} \mathrm{C}$ for $30 \mathrm{~s}$. The primers with high amplification specificity were verified by unique peaks observed in corresponding melting curves. Each plate contained triplicate reactions for each DNA sample. Melting curves were also traced after each assay to confirm that the fluorescence signal had been retrieved from specific PCR products and to ensure the absence of primer dimers. Sterile water was used as the negative control. Parametric data were tested by t-tests and one-way analysis of variance using SPSS 17.0 for Windows software (SPSS Inc, Chicago, USA).

\section{Results}

\section{Identification and phylogenetic analysis of symbionts}

After constructing 16S rRNA gene libraries and RFLP analyses, two different bacterial genera were detected from the $H$. tibetensis. After submitting the sequences to GenBank, about 99 and $97 \%$ of the sequences showed sequence similarities with Rickettsia japonica (GenBank NR074459) and Coxiella-like symbionts from Dermacentor silvarum
(GenBank JN866592), and therefore they were assigned into genera Coxiella (GenBank KU758901 and KU758902) and Rickettsia (GenBank KU758903 and KU758904) and ultimately named as CLS-Ht (Coxiella-like symbiont in $H$. tibetensis) and RLS-Ht (Rickettsia-like symbiont in H. tibetensis). After phylogenetic analysis, the partial $16 \mathrm{~S}$ rRNA gene sequence of CLS-Ht proved to be close to that of the symbiotic Coxiella in Rhipicephalus sanguineus (GenBank D84559), and RLS-Ht clustered with Rickettsia peacockii (GenBank DQ062433) (Fig. 1).

\section{Prevalence of symbionts}

A total of 20 females and 20 males from the field colony were collected and studied by diagnostic PCR and sequencing. The results showed that all the ticks were CLS-Ht and RLS-Ht positive, suggesting that the infection rate of CLS-Ht and RLS-Ht might be $100 \%$ in $H$. tibetensis (Additional file 1: Figure S1).

\section{Vertical transmission of the symbionts}

To test whether transmission of CLS-Ht and RLS-Ht was transovarial or transstadial, samples of eggs, the first generation (F1) larvae, F1 nymphs, F1 females and F1 males were screened. All the tick extracts were infected with CLS-Ht and RLS-Ht, which is consistent with vertical transmission (Additional file 2: Figure S2).

\section{Tissue distribution of the symbionts}

The distribution analysis revealed that CLS-Ht was sepcifically harbored in ovaries and Malpighian tubules, whereas RLS-Ht was harbored in ovaries, Malpighian tubules, salivary glands and midguts of $H$. tibetensis (Additional file 3: Figure S3).

\section{Population dynamics of the symbionts}

The density of CLS-Ht was high in adults but was at a low level in eggs, larvae and nymphs. For RLS-Ht, the results showed a low level in eggs and larvae but an increasing trend was seen from larvae to nymphs, and reached the peak in adults (Figs. 2 and 3). After feeding of larvae and 


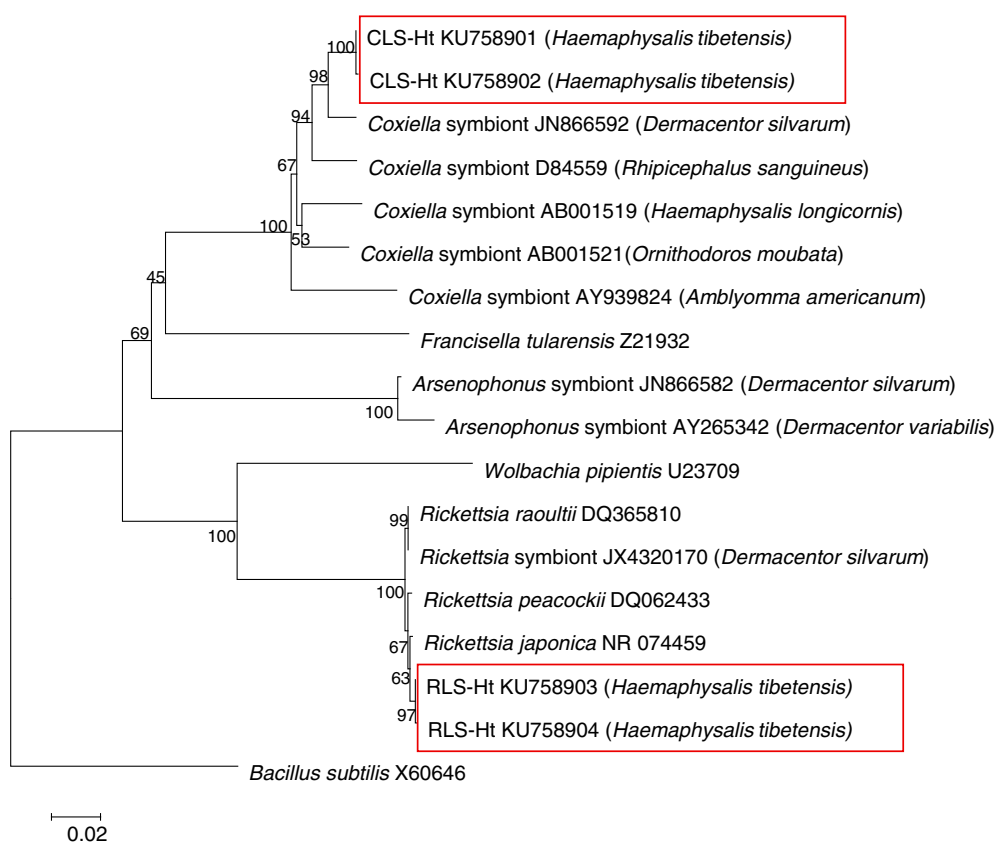

Fig. 1 Neighbor-Joining unrooted phylogenetic tree of CLS-Ht and RLS-Ht symbionts of H. tibetensis and other tick-associated symbionts. The percentage of replicate trees in which the associated taxa clustered together in the bootstrap test (1,000 replicates) is shown next to the branches. Genetic distance was computed using the Kimura 2-parameter method and are in the units of the number of base substitutions per site. Sequence alignments and tree generation were conducted in MEGA6

nymphs, the CLS-Ht was decreased, whereas the RLS-Ht was increased (Fig. 3). When compared between sexes, CLS-Ht abundance was significantly higher in females than in males $\left(t_{(4)}=5.43, P=0.011\right)$, whereas no obvious differences were observed in RLS-Ht between females and males $\left(t_{(4)}=0.92, P=0.41\right)$ (Fig. 4).

In the ovaries, the densities of CLS-Ht were at lower levels in non-engorged stages, and increased on the first day after engorgement but subsequently declined slightly on the $5^{\text {th }}$ day after engorgement (Fig. 5). In Malpighian tubules, CLS-Ht was high one day after engorgement, declined the second day after engorgement, and thereafter the CLS-Ht increased again until the fifth day after engorgement.

The amount of RLS-Ht in ovaries of $H$. tibetensis was significantly higher than in any other organ $\left(F_{(4,21)}=7.19\right.$, $P=0.012$ ), where the highest copy ratio reached 70 and varied randomly with time. In Malphibian tubules, the amount of RLS-Ht showed an elevating trend with copy ratio increasing from 10 to 40 . In midgut, no obvious changes occurred, as the lowest copy ratio seen in the first day of engorgement (which was below 10) remained fairly

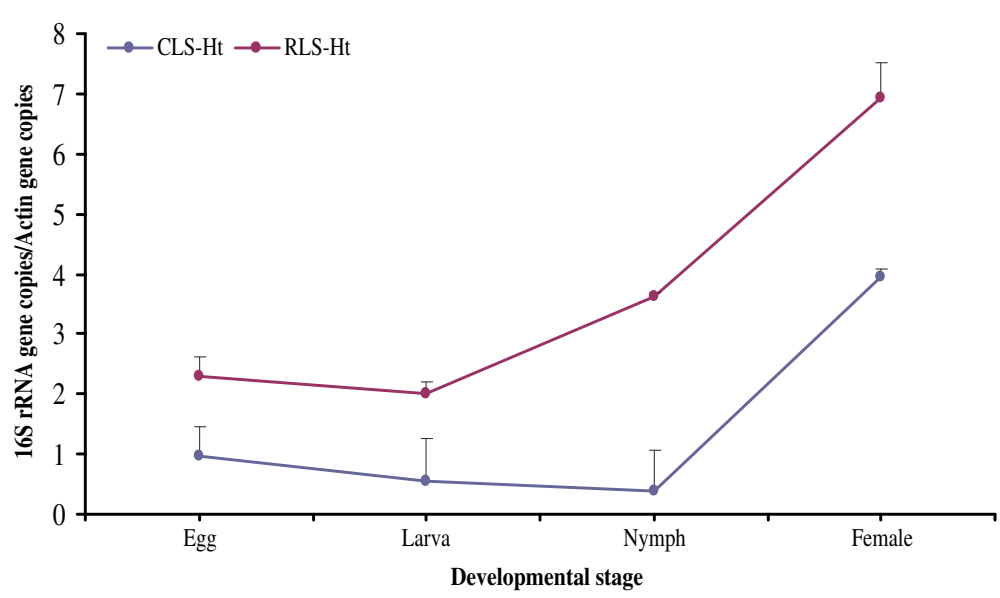

Fig. 2 The density dynamics of CLS-Ht and RLS-Ht with H. tibetensis at different developmental stages. Means and standard errors of means are shown 


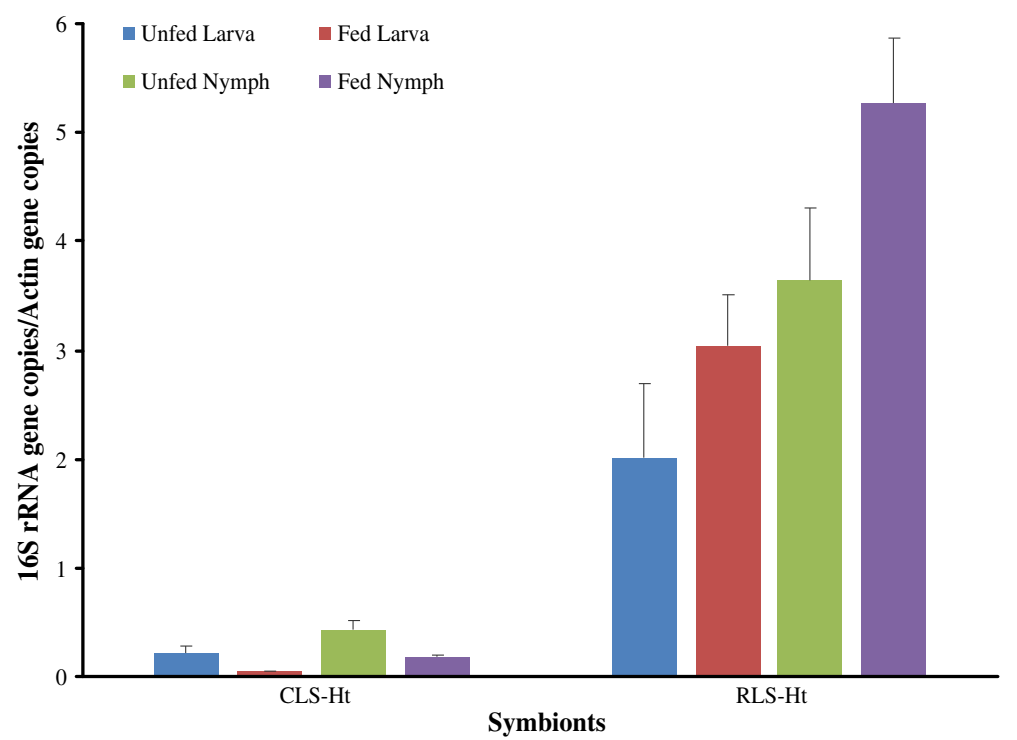

Fig. 3 The quantitative dynamic of CLS-Ht and RLS-Ht in H. tibetensis before and after feeding. Means and standard errors of means are shown

stable afterward at 20 . In the salivary glands, there were no obvious changes with copy ratio consistently below 2 (Fig. 6).

\section{Discussion}

Ticks are notorious for acting as vectors and also serve as reservoirs of a great diversity of mammalian pathogens, hence the microbiome and endosymbionts within ticks have attracted the attention of researchers since each tick species harbors its own unique bacterial community $[3,34]$. In the Tibetan tick $H$. tibetensis, CLS-Ht and RLS-Ht were characterized by constructing $16 \mathrm{~S}$ rRNA libraries and RLFPs. Both CLS-Ht and RLS-Ht showed $100 \%$ infectivity and vertical transmission in $H$. tibetensis; however, differences in tissue-specific distribution was observed. The CLS-Ht mainly infects the ovaries and the Malpighian tubes, which is consistent with the previous observation of Coxiella-like symbionts in $R$. sanguineus and $R$. turanicus ticks [18]. No tissue-specific infectivity was observed for RLS-Ht, a conclusion that is similar with the Rickettsia-like symbiont distribution in $H$. longicornis and D. silvarum found previously [4]. The coinfection of symbionts is common in ticks. Endosymbionts belonging to the genera Rickettsia, Coxiella and Arsenophous have been found in A. americaum where species of all of the three genera showed vertical

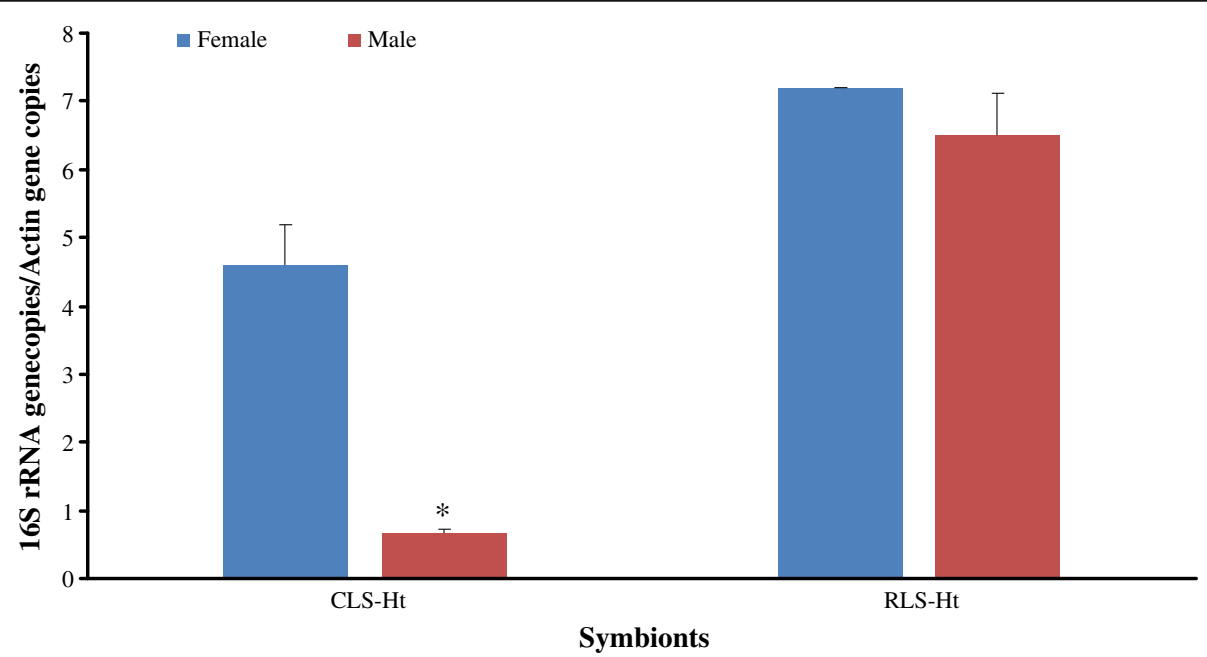

Fig. 4 The quantitative dynamic of CLS-Ht and RLS-Ht in female and male H. tibetensis. Means and standard errors of means are shown, and asterisk indicates a statistical difference in each group $(P<0.05)$ 


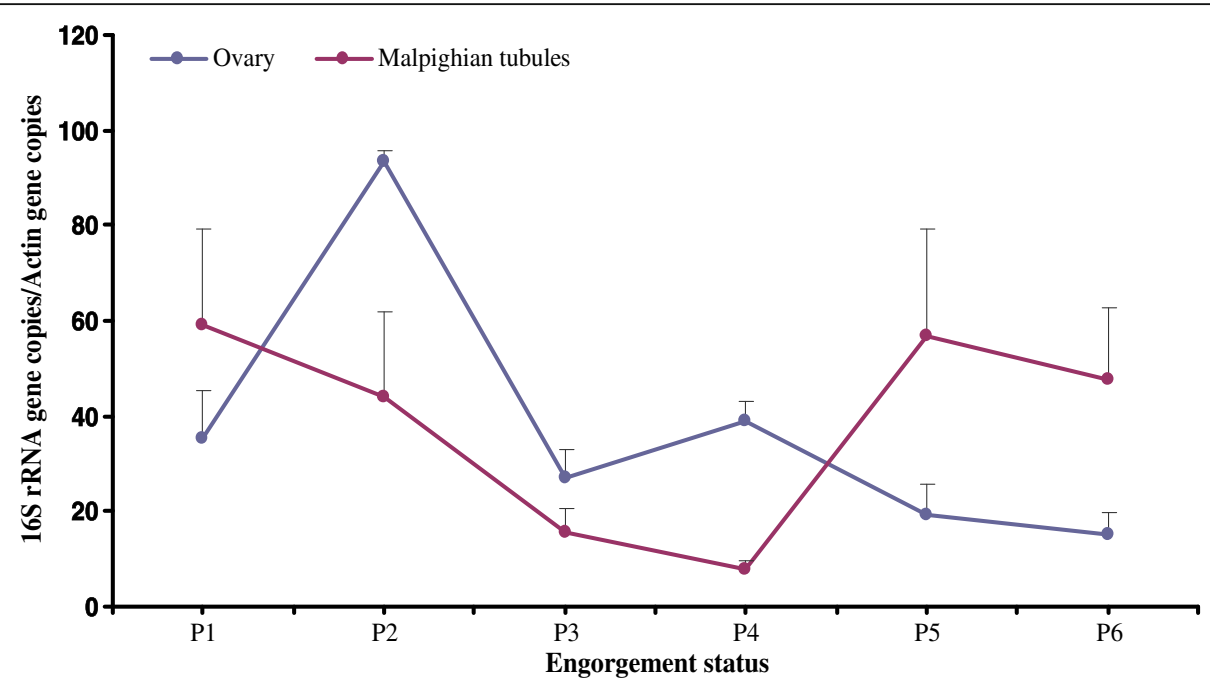

Fig. 5 The dynamics of CLS-Ht in the ovaries and the Malpighian tubules of H. tibetensis. P1-6: 1-6 days post-engorgement. Means and standard errors of means are shown

transmission [35]. The Coxiella-like and Francisella-like symbionts were found coinfected in Ornithodorus moubata [36, 37]. In the tick $R$. turanicus and $R$. sanguineus, both Coxiella-like and Rickettsia-like symbionts were detected, and densities were overall stable throughout the questing season [38].

In the tick $H$. tibetensis, the density of both CLS-Ht and RLS-Ht varied with respect to the developmental stage of the host, showing the highest density in adults. A relatively low density was observed in eggs, larvae and nymphs and the relative stable of density among these developmental stages potentially due to the bottleneck effect during vertical transmission [39]. A similar phenomenon was also observed in the intracellular symbiont "Candidatus Midichloria mitochondrii" harbored in Ixodes ricinus and numerous insect symbionts [40-42]. The narrow bottleneck effect could give rise to more genetic drift in symbiont populations, which would cause further genome erosion and streamlining [43, 44], and increasing evidences have been found in reduced genome of Coxiella-like symbionts in $A$. americanum [21] and $R$. turanicus [22].

A sex-specific distribution of CLS-Ht, with high density in females and low density in males was observed in the tick $H$. tibetensis. Similar results were also observed in the distribution of Coxiella-like endosymbionts in H. hystricis, $H$. lagrangei, H. obesa, H. shimoga [45] and R. turanicus [18]. However, no sex-specific distribution was observed for RLS-Ht in $H$. tibetensis. After feeding of larvae and nymphs, the density of RLS-Ht was increased when compared to the unfed group. Similar results were observed in the amount of "Candidatus Midichloria mitochondrii" in I. ricinus, hence, they were putatively involved in the feeding process of ticks [46]. Both CLS-Ht and RLS-Ht were

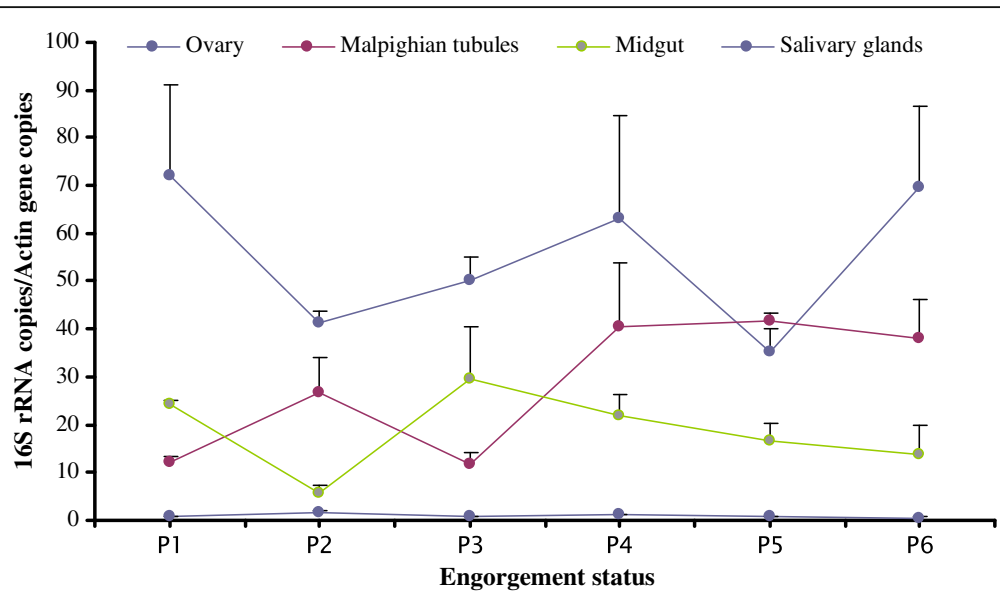

Fig. 6 The dynamics of RLS-Ht in different tissues of H. tibetensis. P1-6: 1-6 days post-engorgement. Means and standard errors of means are shown 
mainly distributed in the ovaries in this study similar to the previous findings for D. silvarum [47]; however, the dynamics of both endosymbionts was different. The CLS-Ht density reached a peak one day after engorgement but subsequently decreased to lower levels, whereas RLS-Ht densities varied randomly while remaining relatively high in ovaries compared to other organs in females.

\section{Conclusions}

Taken together, two novel endosymbionts (CLS-Ht and RLS-Ht) were characterized, both showing a high prevalence and stable vertical transmission in $H$. tibetensis. The described tissue distribution and population dynamics might imply the important functions of these symbionts during the development and reproduction of ticks. Further investigations are required to explore the interactions between CLS-Ht, RLS-Ht and ticks in order to further characterize the effects of the host-pathogen interactions.

\section{Additional files}

Additional file 1: Figure S1. PCR analysis of the prevalence of CLS-Ht and RLS-Ht in H. tibetensis adults. (PPTX $215 \mathrm{~kb}$ )

Additional file 2: Figure S2. PCR analysis of the vertical transmission of CLS-Ht and RLS-Ht in H. tibetensis. (PPTX $68 \mathrm{~kb}$ )

Additional file 3: Figure S3. Detection of infection sites of two symbionts by PCR from different tissues of $H$. tibetensis. (PPTX $66 \mathrm{~kb}$ )

\section{Abbreviations}

CLS-Ht: Coxiella-like symbiont in H. tibetensis; F1: The first generation; RFLP: Restriction fragment length polymorphism; RLS-Ht: Rickettsia-like symbiont in $\mathrm{H}$. tibetensis; RT-qPCR: Real-time quantitative polymerase chain reaction

\section{Acknowledgements}

We would like to thank Professor Kenneth B. Storey and Rasha Al-attar from Carleton University, Canada for English editing. The visit of Zhijun Yu to the Carleton University was generously supported by China Scholarship Council and Hebei Normal University.

\section{Funding}

This work was supported by the National Natural Science Foundation of China (No. 31272372, 31400342, 31672365), Specialized Research Fund for the Doctoral Program of Higher Education of China (No. 20131303130001), the Natural Science Foundation of Hebei Province (C2015205124), and the Natural Science Research Programs of the Educational Department of Hebei Province (BJ2016032).

\section{Availability of data and materials}

The nucleic acid sequences of CLS-Ht and RLS-Ht supporting the conclusions of this article are available in the GenBank (accession numbers: KU758901 and KU758902 for CLS-Ht; KU758903 and KU758904 for RLS-Ht). Other data used in the present study are available from the corresponding author on reasonable request.

\section{Authors' contributions}

RRW and NXL performed the experiments and wrote the manuscript. JNL, TL and ML participated in data collection and analyzed the data. ZJY and JZL conceived and designed the experiments, and reviewed and edited the manuscript. All authors read and approved the final manuscript.

\section{Competing interests}

The authors declare that they have no competing interests.
Consent for publication

Not applicable.

Ethics approval and consent to participate

All protocols for animals were approved by the Institutional Animal Care and Use Committee (IACUC) of Hebei Normal University (permit number: IACUC2014008)

\section{Publisher's Note}

Springer Nature remains neutral with regard to jurisdictional claims in published maps and institutional affiliations.

\section{Author details}

${ }^{1}$ Key Laboratory of Animal Physiology, Biochemistry and Molecular Biology of Hebei Province, College of Life Sciences, Hebei Normal University, Shijiazhuang 050024, China. ${ }^{2}$ Institute of Biochemistry, Department of Biology, Carleton University, 1125 Colonel By Drive, Ottawa, ON K1S 5B6, Canada.

Received: 20 March 2017 Accepted: 15 May 2017

Published online: 25 May 2017

References

1. Sonenshine DE, Roe R. Biology of ticks. 2nd ed. Oxford: Oxford University Press; 2013.

2. Clay K, Fuqua C. The tick microbiome: diversity, distribution and influence of the internal microbial community for a blood-feeding disease vector[C]// Critical needs and gaps in understanding prevention, amelioration, and resolution of Lyme and other tick-borne diseases: the short-term and longterm outcomes. Workshop report. Washington, DC: National Academies Press. 2010.

3. Narasimhan S, Fikrig E. Tick microbiome: The force within. Trends Parasitol. 2015;31:315-23.

4. Liu LM, Liu JN, Liu Z, Yu ZJ, Xu SQ, Yang XH, et al. Microbial communities and symbionts in the hard tick Haemaphysalis longicornis (Acari: Ixodidae) from north China. Parasit Vectors. 2013;6:310.

5. Nakao R, Abe T, Nijhof AM, Yamamoto S, Jongejan F, Ikemura T, Sugimoto C. A novel approach, based on BLSOMs (Batch Learning Self-Organizing Maps), to the microbiome analysis of ticks. ISME J. 2013;7:1003-15.

6. Budachetri K, Browning RE, Adamson SW, Dowd SE, Chao CC, Ching WM, Karim S. An insight into the microbiome of the Amblyomma maculatum (Acari: Ixodidae). J Med Entomol. 2014;51:119-29.

7. Qiu Y, Nakao R, Ohnuma A, Kawamori F, Sugimoto C. Microbial population analysis of the salivary glands of ticks: a possible strategy for the surveillance of bacterial pathogens. PLoS One. 2014;9:e0103961.

8. Carpi G, Cagnacci F, Wittekindt NE, Zhao F, Qi J, Tomsho LP, et al. Metagenomic profile of the bacterial communities associated with Ixodes ricinus ticks. PLoS One. 2011;6:e25604.

9. Menchaca AC, Visi DK, Strey OF, Teel PD, Kalinowski K, Allen MS, Williamson PC. Preliminary assessment of microbiome changes following blood-feeding and survivorship in the Amblyomma americanum nymph-to-adult transition using semiconductor sequencing. PLoS One. 2013;8:e67129.

10. Duron O, Binetruy F, Noël V, Cremaschi J, McCoy KD, Arnathau C, et al. Evolutionary changes in symbiont community structure in ticks. Mol Ecol. 2017; doi:10.1111/mec.14094

11. Dergousoff SJ, Chilton NB. Detection of a new Arsenophonus-type bacterium in Canadian populations of the Rocky Mountain wood tick, Dermacentor andersoni. Exp Appl Acarol. 2010:52:85-91.

12. Ivanov IN, Mitkova N, Reye AL, Hübschen JM, Vatcheva-Dobrevska RS, Dobreva EG, et al. Detection of new Francisella-like tick endosymbionts in Hyalomma spp. and Rhipicephalus spp. (Acari: Ixodidae) from Bulgaria. Appl Environ Microbiol. 2011;77:5562-5.

13. Baldridge GD, Scoles GA, Burkhardt NY, Schloeder B, Kurtti TJ, Munderloh UG. Transovarial transmission of Francisella-like endosymbionts and Anaplasma phagocytophilum variants in Dermacentor albipictus (Acari: Ixodidae). J Med Entomol. 2009;46:625-32.

14. Zhang $X$, Norris DE, Rasgon JL. Distribution and molecular characterization of Wolbachia endosymbionts and filarial nematodes in Maryland populations of the lone star tick (Amblyomma americanum). FEMS Microbiol Ecol. 2011;77:50-6. 
15. Gurfield N, Grewal S, Cua LS, Torres PJ, Kelley ST. Endosymbiont interference and microbial diversity of the Pacific coast tick, Dermacentor occidentalis, in San Diego County, California. PeerJ. 2017;5:e3202.

16. Ahantarig A, Trinachartvanit W, Baimai V, Grubhoffer L. Hard ticks and their bacterial endosymbionts (or would be pathogens). Folia Microbiol. 2013:58:419-28.

17. Raele DA, Galante D, Pugliese N, De Simone E, Cafiero MA. Coxiella-like endosymbiont associated to the "Anatolian brown tick" Rhipicephalus bursa in Southern Italy. Microbes Infect. 2015;17:799-805.

18. Lalzar I, Friedmann Y, Gottlieb Y. Tissue tropism and vertical transmission of Coxiella in Rhipicephalus sanguineus and Rhipicephalus turanicus ticks. Environ Microbiol. 2014;16:3657-68.

19. Duron O, Noël V, McCoy KD, Bonazzi M, Sidi-Boumedine K, Morel O, et al. The recent evolution of a maternally-inherited endosymbiont of ticks led to the emergence of the $\mathrm{Q}$ fever pathogen, Coxiella burnetii. PLoS Pathog. 2015;11:e1004892.

20. Zhong J, Jasinskas A, Barbour AG. Antibiotic treatment of the tick vector Amblyomma americanum reduced reproductive fitness. PLoS One. 2007;2:e405.

21. Smith TA, Driscoll T, Gillespie JJ, Raghavan R. A Coxiella-like endosymbiont is a potential vitamin source for the Lone Star tick. Genome Biol Evol. 2015;7:831-8.

22. Gottlieb Y, Lalzar I, Klasson L. Distinctive genome reduction rates revealed by genomic analyses of two Coxiella-like endosymbionts in ticks. Genome Biol Evol. 2015;7:1779-96.

23. Hunter DJ, Torkelson JL, Bodnar J, Mortazavi B, Laurent T, Deason J, et al. The Rickettsia endosymbiont of Ixodes pacificus contains all the genes of de novo folate biosynthesis. PLoS One. 2015;10:e0144552.

24. Zhang PH, Cao JT, Li YC, Zhang QE. Ultrastructure observation of newly GRD spirochetes isolate in Tibet. In: Zhang QE, editor. Corpus of epidemiological investigation. 2nd ed. Beijing: Publishing House of Academy of Military Medical Sciences of PLA; 1996.

25. Huang XR, Chen J, Chen LY, Wu QL, Yang XX, Li XY, Li DR. Studies on sedimentation coefficient and molecular weight of the orbivirus (Ti3010) isolated from Tibet, China. Bull Acad Milit Med Sci. 2000;23:269-71.

26. Liu M, Li T, Yu ZJ, Gao XH, Zuo CW, Wang RR, et al. Characterization of the life cycle of the tick Haemaphysalis tibetensis under field conditions in Qinghai-Tibet plateau. Exp Appl Acarol. 2016;69:107-15.

27. Hoogstraal H. Haemaphysalis tibetensis sp. n., and its significance in elucidating phylogenetic patterns in the genus (Ixodoidea, Ixodidae). J Parasitol. 1965;51:452-9.

28. Teng GF, Jiang ZJ. Economic insect fauna of China. fasc. 39, Acarina, Ixodidae. Beijing: Science Press; 1991.

29. Jin S, Wang TH, Liu M, Li T, Yang XL, Wang H, et al. Scanning electron microscopy and morphometrics of all parasitic stages of the tick Haemaphysalis tibetensis Hoogstraal, 1965 (Acari: Ixodidae). Syst Appl Acarol. 2016;21:1202-9.

30. Wang TH, Jin $\mathrm{S}$, Liu $M$, Li T, Jia QY, Yang XL, et al. Life cycle of Haemaphysalis tibetensis Hoogstraal, 1965 (Acari: Ixodidae) under laboratory conditions. Syst Appl Acarol. 2017:22:85-90.

31. Liu JN, Yu ZJ, Liu LM, Li NX, Wang RR, Zhang CM, Liu JZ. Identification, distribution and population dynamics of Francisella-like endosymbiont in Haemaphysalis doenitzi (Acari: Ixodidae). Sci Rep. 2016;6:35178.

32. Baldridge GD, Burkhardt NY, Simer JA, Kurtti TJ, Munderloh UG. Sequence and expression analysis of the ompA gene of Rickettsia peacockii, an endosymbiont of the rocky mountain wood tick, Dermacentor andersoni. Appl Environ Microbiol. 2004;70:6628-36.

33. Wilson KH, Blitchington RB, Greene RC. Amplification of bacterial 165 ribosomal DNA with polymerase chain reaction. J Clin Microbiol. 1990;28:1942-6.

34. Van Treuren W, Ponnusamy L, Brinkerhoff RJ, Gonzalez A, Parobek CM Juliano JJ, et al. Variation in the microbiota of Ixodes ticks with regard to geography, species, and sex. Appl Environ Microbiol. 2015;81:6200-9.

35. Clay K, Klyachko O, Grindle N, Civitello D, Oleske D, Fuqua C. Microbial communities and interactions in the lone star tick, Amblyomma americanum. Mol Ecol. 2008;17:4371-81.

36. Reinhardt C, Aeschlimann A, Hecker H. Distribution of Rickettsia-like microorganisms in various organs of an Ornithodoros moubata laboratory strain (Ixodoidae, Argasidae) as revealed by electron microscopy. Zeitschrift fur Parasitenkunde. 1972;39:201-9.
37. Noda H, Munderloh UG, Kurtti TJ. Endosymbionts of ticks and their relationship to Wolbachia spp. and tick-borne pathogens of humans and animals. Appl Environ Microbiol. 1997;63:3926-32.

38. Lalzar I, Harrus S, Mumcuoglu KY, Gottlieb Y. Composition and seasonal variation of Rhipicephalus turanicus and Rhipicephalus sanguineus bacterial communities. Appl Environ Microbiol. 2012;78:4110-6.

39. Kaltenpoth M, Goettler W, Koehler S, Strohm E. Life cycle and population dynamics of a protective insect symbiont reveal severe bottlenecks during vertical transmission. Evol Ecol. 2010;24:463-77.

40. Epis S, Mandrioli M, Genchi M, Montagna M, Sacchi L, Pistone D, Sassera D. Localization of the bacterial symbiont Candidatus Midichloria mitochondtrii within the hard tick Ixodes ricinus by whole-mount FISH staining. Ticks Tick Borne Dis. 2013:4:39-45.

41. Mira A, Moran NA. Estimating population size and transmission bottlenecks in maternally transmitted endosymbiotic bacteria. Microb Ecol. 2002:44:137-43.

42. Hosokawa T, Kikuchi Y, Fukatsu T. How many symbionts are provided by mothers, acquired by offspring, and needed for successful vertical transmission in an obligate insect-bacterium mutualism? Mol Ecol. 2007;16:5316-25.

43. Degnan PH, Lazarus AB, Brock CD, Wernegreen JJ. Host-symbiont stability and fast evolutionary rates in an ant-bacterium association: cospeciation of Camponotus species and their endosymbionts, Candidatus Blochmannia. Syst Biol. 2004:53:95-10.

44. Gil R, Latorre A, Moya A. Bacterial endosymbionts of insects: insights from comparative genomics. Environ Microbiol. 2004:6:1109-22.

45. Arthan W, Sumrandee C, Hirunkanokpun S, Kitthawee S, Baimai V, Trinachartvanit W, Ahantarig A. Detection of Coxiella-like endosymbiont in Haemaphysalis tick in Thailand. Ticks Tick Borne Dis. 2015;6:63-8.

46. Sassera D, Beninati T, Bandi C, Bouman EAP, Sacchi L, Fabbi M, Lo N. 'Candidatus Midichloria mitochondrii', an endosymbiont of the tick Ixodes ricinus with a unique intramitochondrial lifestyle. Int J Syst Evol Microbiol. 2006:56:2535-40.

47. Liu LM, Li LX, Liu JN, Yu ZJ, Yang XH, Liu JZ. Population dynamics of multiple symbionts in the hard tick, Dermacentor silvarum Olenev (Acari: Ixodidae). Ticks Tick Borne Dis. 2015;7:188-92.

\section{Submit your next manuscript to BioMed Central and we will help you at every step:}

- We accept pre-submission inquiries

- Our selector tool helps you to find the most relevant journal

- We provide round the clock customer support

- Convenient online submission

- Thorough peer review

- Inclusion in PubMed and all major indexing services

- Maximum visibility for your research

Submit your manuscript at www.biomedcentral.com/submit 